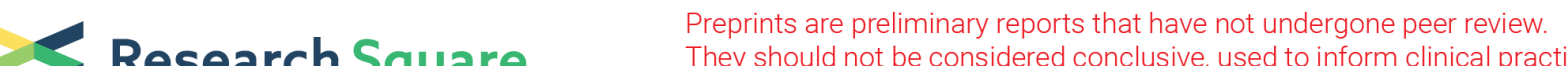 $\begin{array}{ll}\text { Research Square } & \text { They should not be considered conclusive, used to inform clinical practice, } \\ \text { or referenced by the media as validated information. }\end{array}$
}

\section{Brain Activation of College Students in China With Premenstrual Syndrome Localized by BOLD-fMRI}

\section{Dongmei Gao}

Shandong University of Traditional Chinese Medicine

\section{Mingzhou Gao}

Shandong University of Traditional Chinese Medicine

\section{Li An}

Jinan Central Hospital

\section{Yanhong Yu}

Shandong University of Traditional Chinese Medicine

\section{Jieqiong Wang}

Shandong University of Traditional Chinese Medicine

\section{Yanjiao Hou}

Open University of China Press Jinan Branch

\section{Huang Jian}

Zhejiang University

\section{Qiuqi Xu}

Shandong University of Traditional Chinese Medicine

Lvning Ren

Shandong University of Traditional Chinese Medicine

Mingqi Qiao ( $\square$ qmingqi@163.com )

Shandong University of Traditional Chinese Medicine

\section{Research Article}

Keywords: PMS, task state, BOLD-fMRI, block design

Posted Date: July 22nd, 2021

DOI: https://doi.org/10.21203/rs.3.rs-568217/v1

License: (9) This work is licensed under a Creative Commons Attribution 4.0 International License. Read Full License 


\section{Abstract}

\section{Background:}

Most studies on the mechanism behind premenstrual syndrome (PMS) have focused on fluctuating hormones, but little evidence exists regarding functional abnormalities in the affected brain regions of college students. Thus, the aim of this study is to localize PMS's abnormal brain regions by BOLD-fMRI in college students.

\section{Methods:}

Thirteen PMS patients and fifteen healthy control $(\mathrm{HC})$ subjects underwent a BOLD-fMRI scan during the luteal phase induced by depressive emotion pictures. The BOLD-fMRI data were processed by SPM 8 software and rest software based on MATLAB platform. Each cluster volume threshold (cluster) was greater than 389 continuous voxels, and the brain area with single voxel threshold $\mathrm{P}<0.05$ (after correction) was defined as the area with a significant difference. The emotion report form and the instruction implementation checklist were used to evaluate the emotion induced by picture.

\section{Results:}

Compared to the $\mathrm{HC}$, right inferior occipital gyrus, right middle occipital gyrus, right lingual gyrus, right fusiform gyrus, right inferior temporal gyrus, cerebelum_crus1_R囚cerebelum_6_R, culmen, the cerebellum anterior lobe, tuber, cerebellar tonsil of PMS patients were enhanced activation. Sub-lobar》sub-gyral区 extra-nuclear》right orbit part of superior frontal gyrus, right middle temporal gyrus, right Orbit part of inferior frontal gyrus, limbic lobe, right insula, bilateral anterior and adjacent cingulate gyrus, bilateral caudate, caudate head, bilateral putamen, left globus pallidus were decreased activation.

\section{Conclusion:}

Our findings may improve our understanding of the neural mechanisms involved in PMS.

\section{Background}

Premenstrual syndrome (PMS) which causes health problems of women in reproductive age is a disorder, substantially interfering with normal life activities and interpersonal relationships, and related to a woman's menstruation cycle[1]. Established research indicated that the prevalence of PMS was 35.3\% among university students from Sharjah[2], 62.7\% college students in Puducherry[3], and even higher in some regions. PMS brings women diverse symptoms, commonly including affective symptoms such as depression, mood swings and anxiety, physical symptoms such as breast tenderness, bloating; behavioral symptoms such as insomnia, changes in appetite, and difficulty concentrating, affecting their quality of normal life[4]. 
The pathogenesis of PMS is still unclear, but the application of brain imaging technology has facilitated its intrinsic neural mechanism study. There are many technologies related to brain research, including functional near-infrared spectroscopy (fNIRS), neural chip technology, functional magnetic resonance imaging (fMRI), single-photon emission computed tomography (SPECT), magnetoencephalography (MEG), proton magnetic resonance spectroscopy (1H MRS), positron emission tomography (PET) and so on. Among them, the principle of PET and fMRI is to measure the blood flow, that is, the activity of neurons needs to increase the oxygen and glucose in the activation area to meet the needs of blood circulation, which has been widely used[5-7].

After the application of brain imaging technology to PMS research, Qing Liu showed decreased connectivity in the middle frontal gyrus (MFG) and the parahippocampal gyrus (PHG) in PMS patients, as well as increased connectivity in the left medial/superior temporal gyri (MTG/STG) and precentral gyrus within the default mode network (DMN) with FMRI[8]. Hai Liao found increased ReHo mainly in the bilateral precuneus, left inferior temporal cortex (ITC), right inferior frontal cortex (IFC) and left middle frontal cortex (MFC) and decreased ReHo in the right anterior cingulate cortex (ACC) of PMS patients at the luteal phase[9]. Besides, structural magnetic resonance imaging showed increased GM volumes in the precuneus/posterior cingulate cortex (precuneus/PCC) and thalamus, and decreased GM volumes in the insula of PMS patients[10]. At the same time, Demao Deng's researches indicated that PMS patients have greater bilateral amygdalae volumes, and increased FC between the amygdala and certain regions of the frontal cortex, the right temporal pole and the insula, as well as decreased FC between the bilateral amygdalae and the right orbitofrontal cortex and right hippocampus[11]. And Peng Liu found decreased prefrontal-thalamic connectivity and increased posterior parietal-thalamic connectivity in the PMS patients with resting-state functional magnetic resonance imaging (fMRI)[12].

Yet, few studies have reported on the central mechanism of premenstrual syndrome which contains depressive emotion. So our study to observe female students who were troubled with PMS depressive emotion囚in order to explore the brain localization of PMS with the BOLD-fMRI technique.

\section{Methods And Materials}

\subsection{Ethics statement}

This study was approved by the Medicine Ethics Committee of the First Affiliated Hospital of Shandong University of Traditional Chinese Medicine, Shandong, China. All research procedures were conducted in accordance with the Declaration of Helsinki. All participants were apprised of the entire experimental procedure and signed an informed consent form.

\subsection{Subjects}

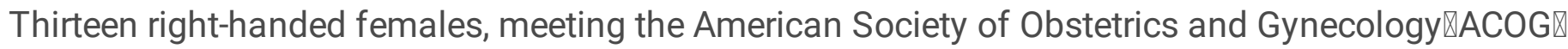
Criteria for Premenstrual Syndrome and depressive emotion, were recruited to participate in the study and were matched with a control group of fifteen comparisons subjects. Fifteen right-handed healthy females, 
university students. Subjects were matched on age $21.13+2.41 \rrbracket$, educational level in each group. In addition, all subjects were administered the Self-rating depression scale (SDS) which aims to determine depression severity and the Toronto Alexithymia scales (TAS-20) to determine the ability to express emotion. Depressed PMS subjects were included with SDS standard score $\geq 50$ and TAS-20 total score $\leqq 66$.Control subjects were included with SDS standard score 50 and TAS- 20 total score $\leqq 66$. All subjects provided written informed consent.

Inclusion criteria were state of mind and quality of sleep, appetite well, consciousness clearly, independent judgment, understanding the purpose of the study and voluntarily with persons; no major diseases, no brain tumors or other brain diseases, no history of taking psychotropic drugs; uncorrected vision or corrected to normal vision; excluding metal objects into the body (including pacemakers, metallic dental materials, wearing braces, etc.); absence history of drug abuse (including the treatment of PMS within three months); absence blood diseases; absence aphasia, unconsciousness, dementia, and other conditions cannot cope with the examine; absence unilateral oophorectomy or abortion, taking contraceptives within 6 months; Data with head motions of more than $3.0 \mathrm{~mm}$ maximum displacement in the $\mathrm{x}, \mathrm{y}$, or $\mathrm{z}$ direction or 2.5 degrees in any angular direction were discarded.

\subsection{Stimuli Paradigm}

During fMRI, negative (NEG) and neutral (NEU) emotion pictures were presented to all subjects, which were held in a block design. Task state contains 2 runs and 6 blocks. Interspersed with emotional pictures in each block were neutral emotional pictures in a pseudo-random order. This ensured the emotional pictures occurred unpredictably. Negative emotion picture stimulus consisted of a 30 sec presentation (each picture was presented for $5 \mathrm{sec}, 6$ pictures in a block) followed by a $30 \mathrm{sec}$ presentation of a neutral face (each picture was presented for $5 \mathrm{sec}, 6$ pictures in a block). Within the emotional stimuli blocks the subject saw 30 negative emotion pictures in a predetermined random order. Within the neutral face blocks the subject saw 30 neutral emotional pictures in a predetermined random order, too. Picture stimuli were presented at a rate of one per echo planar image (EPI) sequence Following each face block there was a block of 30 cross-hair stimuli fixation points $(+)$, presented at the same rate as the emotional pictures, to serve as a control period.

The order of the presentation of the emotional pictures was identical for all subjects across runs. The first run consisted of $+\mathrm{NEG}+\mathrm{NEU}$. The second run was +NEU+NEG. Each run lasted 6 min. Each subject viewed 2 runs. Subjective reports of pictures evoked emotional effects were evaluated by Self-rating depression scale (SDS) and emotional statement and guidance language implementation checklist after the scan. The subjects. Subjective feelings were evaluated by the visual scale test check which scores from 0 (it did not assign to 8 (very strong) points. Higher scores indicated subjects experienced the strength of emotion higher. Subjects must make a careful choice of assessing emotional intensity which may induce different emotions.

Emotional stimuli consisted of negative and neutral emotional pictures which were filtered by the international emotional picture library. Stimuli were presented with the experimental visual stimulus 
program (Electronic Technology in Medicine Co., Ltd., Shenzhen, China), in which each stimulus onset (masked face or crosshair) was triggered directly by a pulse from the scanner. The images were expected onto a computer screen behind the subject's head within the imaging chamber. The screen was viewed by a mirror positioned approximately $8 \mathrm{~cm}$ above the subject's face.

\subsection{Image Acquisition and Data Analysis}

FMRI images were obtained on a 3.0-T MR scanner equipped with a prototype fast gradient system for echo-planar imaging (EPI) at Institute of Medical Imaging of Shandong. Functional images were obtained using an echo-planar imaging sequence with the following parameters: $\mathrm{TE}=35 \mathrm{~ms}$; $\mathrm{TR}=2000 \mathrm{~ms}$; slice thickness $=4 \mathrm{~mm} ;$ gap $=1 \mathrm{~mm}$; flip angle $=90^{\circ} ; \mathrm{FOV}=24 \mathrm{~cm}$; and in-plane resolution $=64 \times 64$.

Functional MRI data were preprocessed using Statistical Parametric Mapping (SPM8). The first 3 volumes of the functional images were discarded because of signal equilibrium and the participants' adaptation to the scanning noise. For each participant, functional images were realigned using leastsquares minimization without higher order corrections for spin history and were normalized to the Montreal Neurological Institute (MNI) template from the structural images. Images were re-sampled to $3 \times 3 \times 3 \mathrm{~mm}^{3}$ and smoothed with a 6 -mm full-width at half maximum.

The data of our study were modelinged generalized linear mode (GJM) using SPM8. So, the data conforms to statistical requirements. Single-sample t-test statistics was used. Volume threshold for each cluster was greater than 389 consecutive voxels, brain regions which single voxel threshold was $P<0.05$ (corrected) Multiple comparison correction for the results was performed by using simulation was defined as statistically significant difference. Double-sample t test was used to analyze the case and control group.

\section{Result}

\subsection{General information}

There were 13 patients in the PMS group and 15 in the HC group. All of them were female college students in Shandong University of Traditional Chinese Medicine, and there were no differences in age, gender, and years of education. All subjects in both groups completed all experimental tasks according to the requirements of this experiment, and all fMRI data collected were valid.

As soon as the subjects of the experiment is finished, they were asked to describe what they had seen of the presented pictures on the emotional statement and guidance language implementation checklist. And to identify depression severity with the Self-rating depression scale (SDS) again. All subjects described disgust after seeing the negative pictures. Depressed PMS subjects $₫$ SDS standard score $\geq 50$.

\subsection{Different activation of brain regions in PMS patients compared with the HC group}


Compared with the HC group, PMS patients show the following enhanced activation of brain areas: right inferior occipital gyrus, right middle occipital gyrus, right lingual gyrus, right fusiform gyrus, right inferior temporal gyrus, cerebelum_crus1_R囚cerebelum_6_R, culmen, cerebellum anterior lobe, tuber, cerebellar tonsil. At the same time, PMS patients show the following decreased activation of brain areas: sub-lobar》 sub-gyral『extra-nuclear囚right orbit part of superior frontal gyrus, right middle temporal gyrus, right Orbit part of inferior frontal gyrus, limbic lobe, right insula, bilateral anterior and adjacent cingulate gyrus, bilateral caudate, caudate head, bilateral putamen and left globus pallidus.

\section{Discussion}

PMDD is a manifestation of underlying depressive disorder which is associated with the inability to regulate emotions in an adaptive manner[13]. And women with PMS have increased mood instability within the seven-day premenstrual phase and at other times as well[14]. An established study indicated that women with PMS appear to have a emotion dysregulation throughout the menstrual cycle[15]. Depression was the most prominent feature of the PMDD diagnosis and should be properly evaluated and treated among women with PMDD[16].

Thus, our findings showed that the activation of the brain area was altered between the PMS and HC groups (Table 1 and Figure 1). The mechanism of PMS is the enhancement of spindle gyrus in occipital lobe and temporal lobe, and activation of right infratemporal gyrus and cerebellum; The activation of sub lobar, sub gyral, extra nucleus, frontal, marginal and basal nuclei was weakened. It shows that the abovementioned brain area function regulation is abnormal before menstruation, and then the emotional, cognitive and attention distribution changes. It can be seen that the enhancement of fusiform gyrus and right infratemporal gyrus and cerebellum activation in occipital and temporal lobes is related to the pathogenesis of the disease.

Our findings are in line with many studies at home and abroad. Frontal lobe is involved in spiritual activities, associated with the individual's emotions. Prefrontal cortex edge, especially the orbitofrontal cortex influenced decision-making and emotional regulation[17]. The right orbitofrontal cortex [18] (middle frontal gyrus) oxygen-dependent reaction weakened when untreated depression patients viewing negative emotions stimuli, this may be related to depression emotion. PMDD patients with luteal phase, the part of the medial prefrontal cortex index levels drops[19], the negative emotional reactions to reduce[20], patients handling negative emotions enhanced, handling of positive emotion weaken, so confirming the existence of behavioral inhibition in the premenstrual phase. PMDD women had higher luteal phase reactivity in the anterior medial prefrontal cortex and dorsolateral prefrontal cortex to negative anticipation than healthy controls[21]. And the medial prefrontal cortex is activated in paradigms of instructed or conditioned fear[22] and is associated with sympathetic nervous system regulation[23]. The cognitive function of PMS patients was relevant with the depression level they felt.

The temporal lobe was related to human emotions and mental activity. The right inferior temporal gyrus[24] has been demonstrated to correlate with cognitive impairment. The fusiform gyrus (medial 
occipitotemporal gyrus) handled the emotional information[25], and the right side is stronger than the left (especially in the processing of negative emotions). The right fusiform gyrus associated with depression[26]. We can know the right fusiform gyrus. Right inferior temporal gyrus is one of the main brain central positions of pathogenesis of PMS, which was discovered for the first time.

The occipital lobe processed visual information, which was linked with temporal lobe. Subcortical nuclei can produce emotion to visual stimuli. Cortical surrounding calcarine belongs to Brodmann area 18,19. And these two regions include the middle occipital gyrus, inferior occipital gyrus, lingual gyrus. BA18 is the second sensory area of processing and integration of visual information. BA19 is able to integrate visual information with other sensory information, and can contact the relevant functional areas of the brain. Compared to normal controls, calcarine cortex of patients in tasks showed deactivation. The result confirmed differences between healthy subjects and patients who were in depression continuously.

Insular is involved in emotional processing, and affected individual decisions[27]. In task state, insula and insular cortex activity of PMDD patients increased significantly in the luteal phase[28]. In task state, our findings showed that the right insula activation decreased, which is a new discovery in premenstrual syndrome research, is not identical with PMDD. That was a new revelation about the inner mechanism of PMS.

Amygdala, hippocampus, and anterior cingulated belongs to Limbic lobe which was related to the emotional functional activities closely. Amygdala of PMDD patients [20] response to negative emotion words stimulation stronger in premenstrual period. Depression scores was correlated to right amygdala response positively in the luteal phase of PMDD[27]. This showed that PMDD patients enhanced processing of negative emotions, weakened positive emotion, reduced limbic lobe activity from top to bottom in premenstrual phase. And there were changes in the hippocampus cortex of PMDD patients[29]. The anterior cingulate was related to the onset of schizophrenia and cognitive disorders[30]. Our study confirmed the limbic played an influential position in PMS, which proved the results of previous related studies.

Basal ganglia involved in higher cognitive functions, for example, memory, emotion. Basal ganglia diseases were linked with cognitive disorders containing Parkinson, Alzheimer's disease, depression[31, 32]. This study found that caudate nucleus, putamen of basal ganglia was associated with the incidence of PMS. This is a new discovery.

Cerebellum was linked with cognitive function[33]. PMDD subjects had greater cerebral grey-matter volume than controls in the posterior cerebellum[34]. Cerebellar activity of PMDD patients increased from follicular phase to late luteal phase[35], especially cerebellar vermis, which was related to emotional deterioration. Our study confirmed it. And our results also showed that culmen, cerebellum anteror lobe, tuber, cerebellar tonsil was associated with PMS closely. In conclusion, Occipital lobe, temporal lobe, cerebellum and other brain regions activation enhanced; Sub-lobar, Sub-Gyral, Extra-Nuclear, frontal, limbic, cingulate cortex, basal ganglia activation weakened. Those brain regions were inextricably linked with the pathogenesis of PMS. 


\section{Conclusions}

In summary, PMS's abnormal brain regions were localized by BOLD-fMRI in college students, indicating pathological changes in brain. But these new findings need to be verified and reappeared through larger sample size and application of animal models in the future.

\section{Abbreviations}

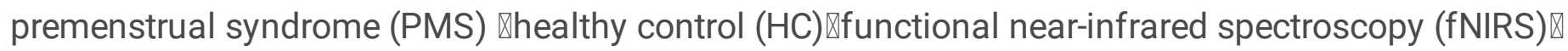
functional magnetic resonance imaging (fMRI) $\llbracket$ single-photon emission computed tomography (SPECT) $\llbracket$ magnetoencephalography (MEG) \proton magnetic resonance spectroscopy (1H MRS)『positron emission

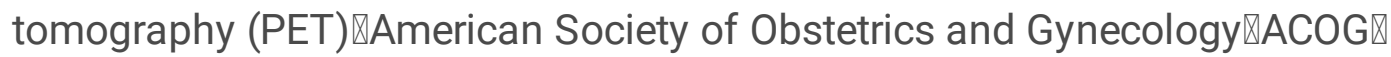

\section{Declarations}

\section{Ethics approval and consent to participate}

This study was approved by the Medicine Ethics Committee of the First Affiliated Hospital of Shandong University of Traditional Chinese Medicine, Shandong, China. All research procedures were conducted in accordance with the Declaration of Helsinki. All participants were apprised of the entire experimental procedure and signed an informed consent form.

\section{Consent for publication}

Not applicable

\section{Availability of data and material}

The datasets used and/or analysed during the current study are available from the corresponding author on reasonable request.

\section{Competing interests}

All the authors declare no conflicts of interest.

\section{Funding}

This research was funded by National Science and Technology Major Project "Key New Drug Creation and Manufacturing Program" of China under Grant SQ2017ZX091064; National Natural Science Foundation of China under Grant 81001484 and 81473558; National ministry of science and technology project 973 of China under Grant 2011CB505102; Key Program of National Natural Science Foundation of China under Grant 30930110.

\section{Authors' contributions}


Dongmei Gao and Qiao MQ designed the study. Mingzhou Gao, Yanjiao Hou, and Li An performed the experiment. Qiuqi Xu and Lvning Ren helped the data collection. Jian Huang undertook the statistical analyses. Mingzhou Gao and Dongmei Gao wrote the draft and revised it. Yanhong Yu and Jieqiong Wang provided key assistance. All authors contributed to and have approved the final manuscript.

\section{Acknowledgements}

We thank all members of Research and Innovation team of Emotional Diseases and Syndromes in Shandong University of Traditional Chinese Medicine for assistance.

\section{References}

1. Yonkers, K.A., P.M.S. O'Brien, and E. Eriksson, Premenstrual syndrome. Lancet (London, England), 2008. 371(9619): p. 1200-1210.

2. Hashim, M.S., et al., Premenstrual Syndrome Is Associated with Dietary and Lifestyle Behaviors among University Students: A Cross-Sectional Study from Sharjah, UAE. Nutrients, 2019. 11(8).

3. Bhuvaneswari, K., P. Rabindran, and B. Bharadwaj, Prevalence of premenstrual syndrome and its impact on quality of life among selected college students in Puducherry. The National medical journal of India, 2019. 32(1): p. 17-19.

4. Mishra, S. and R. Marwaha, Premenstrual Dysphoric Disorder, in StatPearls. 2020: Treasure Island (FL).

5. Heeger, D.J. and D. Ress, What does fMRI tell us about neuronal activity? Nature Reviews Neuroscience, 2002. 3(2): p. 142-151.

6. Bandettini, P., Functional MRI today. International journal of psychophysiology : official journal of the International Organization of Psychophysiology, 2007. 63(2): p. 138-145.

7. Phan, K.L., et al., Functional neuroanatomy of emotion: a meta-analysis of emotion activation studies in PET and fMRI. Neurolmage, 2002. 16(2): p. 331-348.

8. Liu, Q., et al., Abnormal Resting-State Connectivity at Functional MRI in Women with Premenstrual Syndrome. Plos One, 2015. 10(9).

9. Liao, H., et al., Abnormal Spontaneous Brain Activity in Women with Premenstrual Syndrome Revealed by Regional Homogeneity. Frontiers in human neuroscience, 2017. 11: p. 62.

10. Liu, P., et al., Altered brain structure in women with premenstrual syndrome. Journal of affective disorders, 2018. 229: p. 239-246. 
11. Deng, D., et al., Larger volume and different functional connectivity of the amygdala in women with premenstrual syndrome. European radiology, 2018. 28(5): p. 1900-1908.

12. Liu, P., et al., Thalamocortical dysconnectivity in premenstrual syndrome. Brain Imaging Behav, 2019. 13(3): p. 717-724.

13. Reuveni, l., et al., Emotional regulation difficulties and premenstrual symptoms among Israeli students. Arch Womens Ment Health, 2016. 19(6): p. 1063-1070.

14. Bowen, R., et al., Mood instability in women with premenstrual syndrome. Journal of obstetrics and gynaecology Canada : JOGC = Journal d'obstetrique et gynecologie du Canada : JOGC, 2011. 33(9): p. $927-934$.

15. Wu, M., et al., Emotion Dysregulation of Women with Premenstrual Syndrome. Scientific reports, 2016. 6: p. 38501.

16. Ko, C.H., et al., Depression, irritability, and anxiety in women with premenstrual dysphoric disorder. Int J Psychiatry Med, 2013. 46(1): p. 39-55.

17. Berridge, K.C. and M.L. Kringelbach, Neuroscience of affect: brain mechanisms of pleasure and displeasure. Curr Opin Neurobiol, 2013. 23(3): p. 294-303.

18. Feeser, M., et al., Context insensitivity during positive and negative emotional expectancy in depression assessed with functional magnetic resonance imaging. Psychiatry research, 2013. 212(1): $\mathrm{p}$. 28-35.

19. Batra, N.A., et al., Proton magnetic resonance spectroscopy measurement of brain glutamate levels in premenstrual dysphoric disorder. Biol Psychiatry, 2008. 63(12): p. 1178-84.

20. Protopopescu, X., et al., Toward a functional neuroanatomy of premenstrual dysphoric disorder. Journal of Affective Disorders, 2008. 108(1-2): p. 87-94.

21. Gingnell, M., et al., Premenstrual dysphoric disorder and prefrontal reactivity during anticipation of emotional stimuli. Eur Neuropsychopharmacol, 2013. 23(11): p. 1474-83.

22. Etkin, A. and T.D. Wager, Functional neuroimaging of anxiety: a meta-analysis of emotional processing in PTSD, social anxiety disorder, and specific phobia. Am J Psychiatry, 2007. 164(10): p. 147688.

23. Critchley, H.D., C.J. Mathias, and R.J. Dolan, Neuroanatomical basis for first- and second-order representations of bodily states. Nature neuroscience, 2001. 4(2): p. 207-212.

24. Nakatsuka, M., et al., Content of delusional thoughts in Alzheimer's disease and assessment of content-specific brain dysfunctions with BEHAVE-AD-FW and SPECT. Int Psychogeriatr, 2013. 25(6): p. 
939-48.

25. Monroe, J.F., et al., The fusiform response to faces: explicit versus implicit processing of emotion. Hum Brain Mapp, 2013. 34(1): p. 1-11.

26. Surguladze, S., et al., A differential pattern of neural response toward sad versus happy facial expressions in major depressive disorder. Biol Psychiatry, 2005. 57(3): p. 201-9.

27. Gingnell, M., et al., Menstrual cycle effects on amygdala reactivity to emotional stimulation in premenstrual dysphoric disorder. Horm Behav, 2012. 62(4): p. 400-6.

28. Harle, K.M., et al., The neural mechanisms of affect infusion in social economic decisionmaking: a mediating role of the anterior insula. Neuroimage, 2012. 61(1): p. 32-40.

29. Jeong, H.G., et al., Gray matter abnormalities in patients with premenstrual dysphoric disorder: an optimized voxel-based morphometry. Journal of Affective Disorders, 2012. 140(3): p. 260-267.

30. Yan, H., et al., Functional and anatomical connectivity abnormalities in cognitive division of anterior cingulate cortex in schizophrenia. PLoS One, 2012. 7(9): p. e45659.

31. Jokinen, P., et al., Cognitive slowing in Parkinson's disease is related to frontostriatal dopaminergic dysfunction. Journal of the neurological sciences, 2013. 329(1-2): p. 23-28.

32. Tsamis, K.I., et al., Glutamate receptors in human caudate nucleus in normal aging and Alzheimer's disease. Curr Alzheimer Res, 2013. 10(5): p. 469-75.

33. Villanueva, R., The cerebellum and neuropsychiatric disorders. Psychiatry Research, 2012. 198(3): p. 527-532.

34. Berman, S.M., et al., Elevated gray matter volume of the emotional cerebellum in women with premenstrual dysphoric disorder. J Affect Disord, 2013. 146(2): p. 266-71.

35. Rapkin, A.J., et al., Neuroimaging evidence of cerebellar involvement in premenstrual dysphoric disorder. Biol Psychiatry, 2011. 69(4): p. 374-80.

\section{Tables}

Table 1 Double-sample T-test about negative emotion pictures subtracted neutral emotion pictures of case and control group

$\mathrm{P}<0.05$

\section{Figures}




\begin{tabular}{|c|c|c|c|c|c|c|c|}
\hline Lobe & Region & Side & $\begin{array}{l}\text { Signal } \\
\text { peak }\end{array}$ & Voxel & $\mathrm{T}$ & BA & AAL \\
\hline \multirow[t]{5}{*}{$\begin{array}{l}\text { Prefrontal } \\
\text { lobe }\end{array}$} & $\begin{array}{l}\text { orbit part of superior frontal } \\
\text { gyrus }\end{array}$ & Right & $\begin{array}{lll}-12 & 6 & -9\end{array}$ & 105 & -4.3294 & & $\sqrt{ }$ \\
\hline & Middle Temporal Gyrus & Right & $-126-9$ & 67 & -4.3294 & & $\sqrt{ }$ \\
\hline & $\begin{array}{l}\text { Orbit part of inferior frontal } \\
\text { gyrus }\end{array}$ & Right & $\begin{array}{lll}-12 & 6 & -9\end{array}$ & 57 & -4.3294 & & $\sqrt{ }$ \\
\hline & Rectal Gyrus & Left & $\begin{array}{lll}-12 & 6 & -9\end{array}$ & 42 & -4.3294 & & $\sqrt{ }$ \\
\hline & Rectal Gyrus & Right & $\begin{array}{lll}-12 & 6 & -9\end{array}$ & 31 & -4.3294 & & $\sqrt{ }$ \\
\hline \multirow{3}{*}{$\begin{array}{l}\text { Occipital } \\
\text { lobe }\end{array}$} & Inferior Occipital Gyrus & Right & $\begin{array}{l}39-75 \\
-18\end{array}$ & 111 & 3.7694 & & $\sqrt{ }$ \\
\hline & Middle Occipital Gyrus & Right & $\begin{array}{l}39-75 \\
-18\end{array}$ & 34 & 3.7694 & & $\sqrt{ }$ \\
\hline & Lingual Gyrus & Right & $\begin{array}{l}39-75 \\
-18\end{array}$ & 12 & 3.7694 & & $\sqrt{ }$ \\
\hline \multirow{2}{*}{$\begin{array}{l}\text { Temporal } \\
\text { lobe }\end{array}$} & Fusiform Gyrus & Right & $\begin{array}{l}39-75 \\
-18\end{array}$ & 104 & 3.7694 & & $\sqrt{ }$ \\
\hline & Inferior Temporal Gyrus & Right & $\begin{array}{l}39-75 \\
-18\end{array}$ & 28 & 3.7694 & & $\sqrt{ }$ \\
\hline Insula & Insula & Right & $\begin{array}{lll}-12 & 6 & -9\end{array}$ & 22 & -4.3294 & -- & -- \\
\hline Limbic lobe & & & $\begin{array}{lll}-12 & 6 & -9\end{array}$ & 142 & -4.3294 & -- & -- \\
\hline \multirow[t]{9}{*}{ Cingulate } & $\begin{array}{l}\text { anterior and adjacent } \\
\text { cingulate gyrus }\end{array}$ & & $\begin{array}{lll}-12 & 6 & -9\end{array}$ & 21 & -4.3294 & & $\sqrt{ }$ \\
\hline & $\begin{array}{l}\text { anterior and adjacent } \\
\text { cingulate gyrus }\end{array}$ & Right & $-126-9$ & 26 & -4.3294 & & $\sqrt{ }$ \\
\hline & Caudate & Left & $\begin{array}{lll}-12 & 6 & -9\end{array}$ & 107 & -4.3294 & & $\sqrt{ }$ \\
\hline & Caudate Head & & $-126-9$ & 78 & -4.3294 & -- & -- \\
\hline & Putamen & & $-126-9$ & 59 & -4.3294 & -- & -- \\
\hline & Caudate & Right & $-126-9$ & 45 & -4.3294 & -- & -- \\
\hline & Putamen & & $-126-9$ & 43 & -4.3294 & -- & -- \\
\hline & Putamen & Right & $-126-9$ & 24 & -4.3294 & -- & -- \\
\hline & Globus Pallidus & Left & $-126-9$ & 23 & -4.3294 & -- & -- \\
\hline \multirow[t]{2}{*}{ Cerebellar } & Cerebelum_Crus1_R & & $\begin{array}{l}39-75 \\
-18\end{array}$ & 123 & 3.7694 & & $\sqrt{ }$ \\
\hline & Cerebelum_6_R & & $39-75$ & 60 & 3.7694 & & $\sqrt{ }$ \\
\hline
\end{tabular}




\begin{tabular}{lllllll|}
\hline Culmen & $\begin{array}{l}39-75 \\
-18\end{array}$ & 54 & 3.7694 & -- & -- \\
\hline Cerebellum Anterior Lobe & $39-75$ & 54 & 3.7694 & -- & -- \\
& -18 & & & & \\
\hline Tuber & $39-75$ & 35 & 3.7694 & -- & -- \\
\hline Cerebellar Tonsil & -18 & & & & \\
\hline Sub-lobar & $39-75$ & 29 & 3.7694 & -- & -- \\
\hline Sub-Gyral & -18 & & & -4.3294 & -- & -- \\
\hline Extra-Nuclear & -12 & -9 & 289 & -9.3294 & -- & -- \\
\hline
\end{tabular}

A

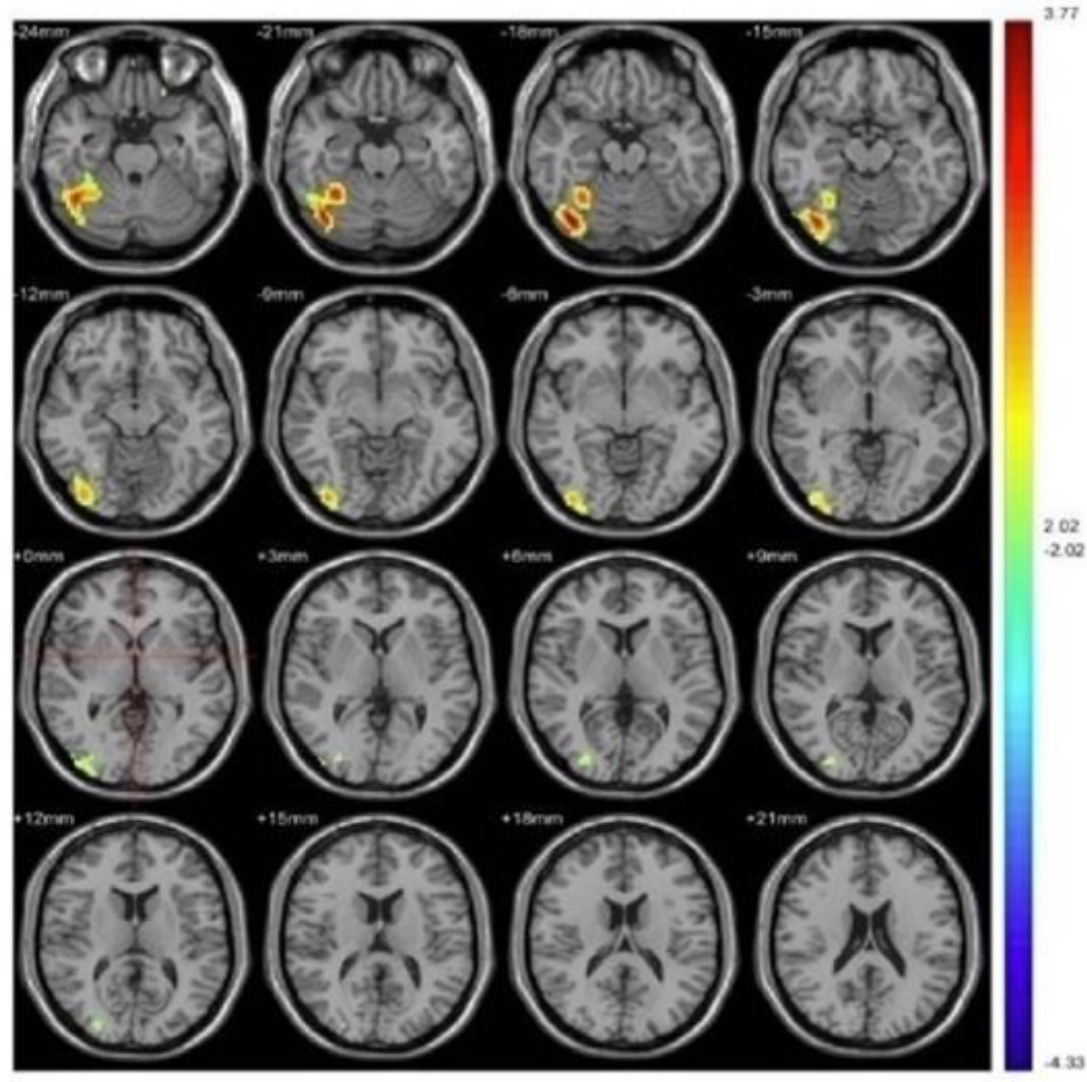

B

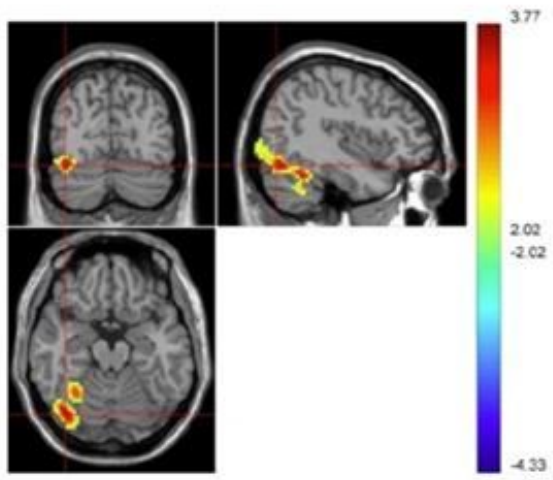

C

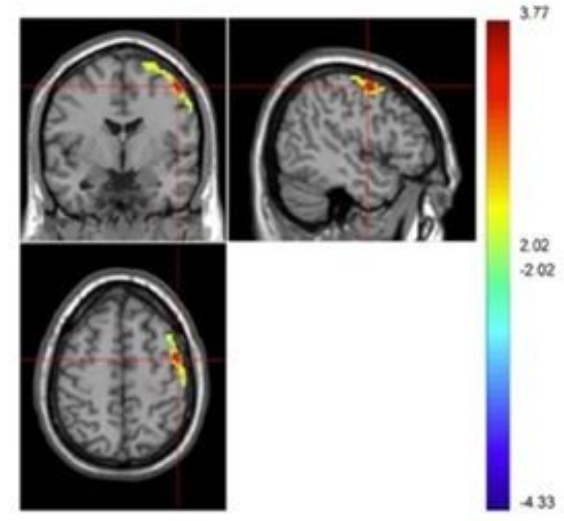

Figure 1

shows the difference of brain regions between the PMS patients and the $\mathrm{HC}$ group under the condition of negative emotion picture minus neutral picture. A show he overall activation of the brain area by the negative emotion picture task in PMS patients; $\mathrm{B}$ shows bilateral temporal lobe; $\mathrm{C}$ shows bilateral 
precuneus, posterior cingulate and cuneiform; $D$ shows the brain areas that are activated in the motor areas. Red indicates that the activation of brain regions in PMS patients is enhanced, while blue indicates that the activation is decreased 